\title{
Is Metal Contamination A Health Risk In Study Subjects From Urban Vadodara?
}

\author{
SUNEETA CHANDORKAR ${ }^{*}$, PRIYANKA BAJAJ and PRACHI DEOTA
}

Department of Foods and Nutrition, The Maharaja Sayajirao University of Baroda, Vadodara- 390002, India.

http://dx.doi.org/10.12944/CWE.10.1.16

(Received: November 30, 2014; Accepted: January 20, 2015)

\begin{abstract}
Metal contamination of food is a major food safety concern emerging at global as well as national level. Air, water and soil are the major routes through which metals enter the food chain. Gujarat being the second most industrialized state of India and Vadodara having the highest number of chemical factories in Gujarat is at a higher risk of metal toxicity. Research has also been done to analyze metal contamination of raw food hence in the present study investigations were done on metal contamination of total diets. The objective was to analyze each meal of the day and water sample of the subjects for metal content by duplicate diet method. Duplicate diets, which included separate food samples for each food groups and drinking water were collected from sixty subjects from free living population of Vadodara. Food samples were digested using wet digestion method. In all 250 foods and 24 water samples were analyzed for metals namely, Aluminum, Arsenic, Cadmium, Mercury, Nickel and Lead using ICP-MS. Amongst all food groups pulse based product had highest contamination of Aluminum, Cadmium and Lead. Nickel contamination was highest in eggs. Arsenic contamination was highest in other items. Maximum Aluminum, Arsenic and Nickel in diets were contributed from cereals whereas maximum Lead and Cadmium was contributed from pulses in the diets. Aluminum, Lead, Cadmium and Nickel were in higher amounts in foods compared to their cutoffs. There is therefore an urgent need to look into possible factors for the high presence of metals in food and take remedial measures to reduce metal contamination.
\end{abstract}

Key words: Metal toxicity, Vadodara, Water, Total diets, Home -based diet, Institutionalized diets.

\section{INTRODUCTION}

Metals are elements that form cations when compounds of it are in solution and oxides of the elements form hydroxides rather than acids in water. The contamination of metals like Lead, Mercury and Arsenic has been found to be detrimental to all the organisms. Metals are released through various sources leading to the contamination of air, water and soil. Metals are non-biodegradable and non-thermo degradable; they enter the human body system through the food chain and accumulate in various organs resulting in various lethal effects (Khan et al, 2009). Metals enter the human body principally through two major routes, i.e. ingestion and respiration (Zhuan et al, 2008). Ingestion is major route of entry for metals in the body, accounting for almost $75 \%$ of the total metal load. Metals are introduced in the food web at various levels through various sources. Contamination starts right at the bottom of the food chain. Metals enter the food chain through the contaminated soil and whereas soil contamination is a consequence of use of effluent water for farming purpose.

Soil contamination leads to the contamination of the plants as they absorb these metals from the land they are grown in. These plants 
when expended by the higher organism leads to intake of metals from food. The latest agronomic practices like use of pesticides; chemical fertilizers etc. are yet another source of incorporating metals in the soil and crop (Pandey \& Pandey, 2009). Metals are engrossed from the air and it is a known fact that due to amplified industrialization the atmosphere is heavily loaded with metals like Arsenic, Zinc, Mercury, etc. Use of unleaded petrol leads to escalated Lead load of the atmosphere. Areas around smelters are found to be worryingly polluted with Zinc. In such a situation these metals can enter human vital organs via respiratory route (Pandey \& Pandey, 2009). Ingestion is a major route of entry of metals and there is variation even amongst the various types of foods in the level of their metal contamination.

Metal contamination if talked about in perspective to Gujarat there are a lot of explanations and indications as to how this problem is aggravating with time. Industrial background of Gujarat noticeably displays that the composition of industries have moved from the textile manufacturing units to chemical product, petrochemicals, pharmaceuticals, metal and metal products, plastics, etc. Numerous cohorts in the state have stated that there is pervasive air, soil and surface and underground water contamination. All these issues in totality pose very threating circumstances in Gujarat as heavy metal contamination is high and is aggravating day by day (Shah \& Mehta, 2003).

Thus rapid industrialization of Gujarat poses a risk to human health due to metal contamination. Lack of legislation leads to release of effluent that contaminates ground water. Agricultural practices are focusing on bringing out higher yield at the cost of metal contamination. Thus there is enough evidence on likelihood of high metal contamination of foods and the same needs to be quantified. Therefore, the present study aimed at estimating the intake of metals (Aluminum, Arsenic, Cadmium, Mercury, Nickel and Lead) across age groups on cooked food basis.

Consequently the broad objective of the study was:

- $\quad$ To assess the health risk due to metal contamination of food
The Specific objectives of the study were as follows:

- To collect information on dietary habits and conduct anthropometry in the study population.

- To analyze each meal of the day and water sample of the subjects for metal content by duplicate diet method.

\section{METHODS AND MATERIALS}

The literature reviewed showed that the commonly found metals in food are Aluminum, Cadmium, Nickel, Arsenic, Lead and Mercury and therefore Aluminum, Cadmium, Mercury, Arsenic and Lead were analyzed in food and water samples. Table 1 shows the experimental design and table 2 depicts the methodology.

\section{Total Diet Study}

A total diet study consists of purchasing at retail level foods commonly consumed, processing them for consumption, often combining the foods into food composites or aggregates, homogenizing them, and analyzing them for toxic chemicals and certain nutrients.

\section{Duplicate Diet}

The duplicate diet approach is a direct sampling technique in which an exact duplicate of food being consumed is obtained and analyzed. (Saipan\& Ruangwises,2009)

\section{Collection of food and water samples}

The subjects were given polyethylene bags for the collection of their duplicate diet for one day. The subjects were given a proforma in which information was also given regarding the study and information as to how the food samples were to be segregated in various bags. The subjects were also instructed to list down food items consumed by them in the proforma. Six bags for food samples along with one acid washed bottle were given to the subjects for the collection of their diet in the following fashion. Bag one was given for cereal based products, bag two for pulse based products, bag three for fruits and vegetables, bag four for milk and milk products and bag five and six were given for any other items consumed in the day. The acid washed bottle was given for the drinking water sample. 
Sixty subjects from free living population of urban Vadodara were enrolled for the study. 10 males and 10 female subjects were taken for each each category namely adolesecents, adults and elderly population.

\section{Sample preparation}

The subjects were asked to store their duplicate diets in the refrigerator by keeping cereal and cereal based products, milk and milk products, fruits and vegetables, pulses and pulse based products and other items separately. Weight of the sample of each food group of the diets was measured on the electronic weighing machine and the weights of the various food samples were recorded. Further the food samples were blenderized for homogenization of the samples. Three grams of each food sample was weighed and taken in Kjeldahl flask. Then acid mixture of 3:2:1 of Nitric acid, Perchloric acid and Sulphuric acid was prepared and $25 \mathrm{ml}$ of this mixture was poured carefully along the walls of the flask in order to wash away any material adhering to the neck of flask. A clean acid washed glass bead was added to the flask to prevent bumping of the contents and the mixture was kept in the Fume cupboard till a clear solution was obtained (which was approximately 3-4 hours). The sample was then cooled and volume of all the samples was made to $10 \mathrm{ml}$ using deionized water. The digested food and drinking water samples were filtered through Whatman filter paper number 40 , so as to remove any dust particle. The samples were properly coded and immediately transferred to acid washed polyethylene bottles of food grade and stored in dark and cool place till further analysis. (Raghuramulu, Nair, \& Kalyanasundaram,2003). The study was approved by Departmental Medical Ethics Committee (F.C.Sc./FND/ME/76)

Table 1: Phase wise experimental design of the study

\begin{tabular}{|c|c|}
\hline Phase 1 & $\begin{array}{l}\text { Enrolling the subjects and collection } \\
\text { of food and water sample and basic } \\
\text { information. }\end{array}$ \\
\hline hase & Preparation of the sample. \\
\hline Phase 3 & Estimation of metals in the samples. \\
\hline
\end{tabular}

\section{RESULTS AND DISCUSSIONS}

The results are presented and discussed in accordance with the objectives of the present study.

\section{Metal content in various food groups}

The metal content of these food groups is given in table 3.

The analyzed diets showed highest contamination with Aluminum, followed by Nickel, Lead, Arsenic, Cadmium and negligible amount of Mercury was detected. Data revealed that the pulses were found to be the most contaminated food group in the diets of the study population. Pulses had the highest contamination levels of Aluminum, Cadmium and Lead. On the other hand other items like flax seeds, laddoes and candies had the highest level of arsenic contamination. Fruits and vegetables were found to have negligible amount of Mercury, while it was absent in all the other food groups. Eggs were found to be least contaminated with metals but had the highest levels of Nickel contamination.

Comparison of metal contamination in home based and institutionalized diets. Out of the 60 subjects 21 were having institutionalized food and 39 were home based diets. The Aluminium, Arsenic, Cadmium, Nickel and Lead content of institutionalized and home based diets is presented in Figure 1-5.

Figure 1 shows the concentration of Aluminum in various food groups in the home based and institutionalized diets of the study population. Aluminum content was higher in cereal based products, milk and milk products, pulse based

Table 2: Methodology used to conduct the study

\begin{tabular}{ll}
\hline Parameter & Tool \\
\hline Total Diet Study & $\begin{array}{l}\text { Duplicate Diet Method } \\
\text { Digestion of Food }\end{array}$ \\
$\begin{array}{ll}\text { Wamples } \\
\text { Estimation of the metal } \\
\text { content }\end{array}$ & $\begin{array}{l}\text { Inductively coupled } \\
\text { plasma mass } \\
\text { Spectrometry }\end{array}$ \\
\hline
\end{tabular}


products and eggs in the institutionalized diets. While in the home based diets Aluminum is higher in fruits and vegetables, other items and nuts. The Aluminum content was highest in pulse based products in the diets of institutionalized subjects and lowest levels of Aluminum were detected in nuts. The Aluminum contamination in pulses was detected to be almost four times in the institutionalized diets as compared to the home based diets. Aluminum content of cereal and cereal product and milk and milk products was found to be more than double in institutionalized diets as compared to the home cooked diets. On the other hand the other items were three times more contaminated with Aluminum in the home cooked diets as compared to the institutionalized diets. In fruits and vegetables and eggs the contamination of Aluminum was similar in both home cooked and institutionalized diets.

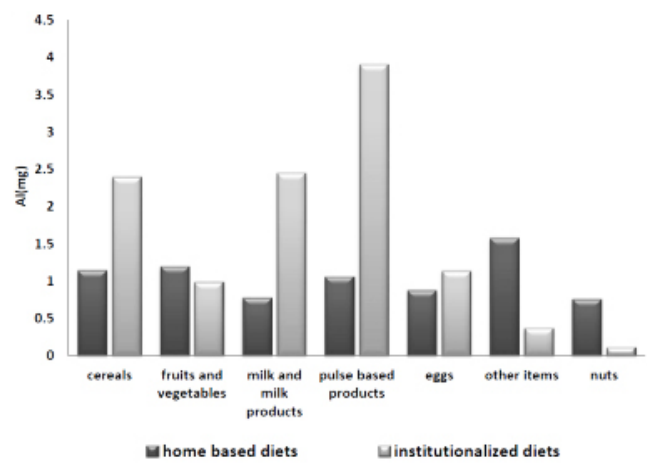

Fig. 1: Comparison of Concentration of Aluminum ( $\mathrm{mg} / 100 \mathrm{~g}$ of cooked food) in home based and institutionalized diets
Figure 2 presents the Arsenic contamination in cereal based products, fruits and vegetables, milk and milk products and pulse based products was detected to be higher in the diets of the institutionalized subjects as compared to the home cooked diets, though the difference was not significant. Arsenic contamination was higher in other items and nuts in the home cooked diets.

In the institutionalized diets pulse based products and nuts had higher levels of Cadmium contamination as compared to the home based diets (Figure 3 ). In cereal based products, fruits and vegetables, milk and milk products, eggs and other items Cadmium concentration was higher in the home based diets as compared to the institutionalized diets. The Cadmium content of

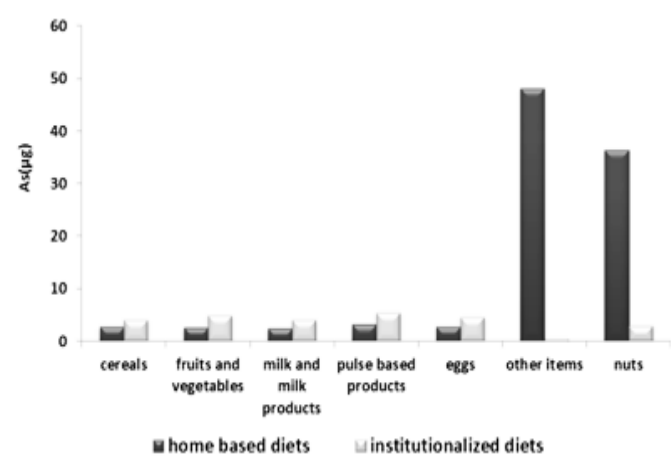

Fig. 2: Comparison of Concentration of Arsenic ( $\mu \mathrm{g} / 100 \mathrm{~g}$ of cooked food) in home based and institutionalized diets

Table 3: Concentration of individual metals in $\mu \mathrm{g} / 100 \mathrm{~g}$ of food groups (cooked basis)

\begin{tabular}{lccccccc}
\hline Food Groups & Al & As & Cd & $\mathbf{H g}$ & $\mathbf{N i}$ & $\mathbf{P b}$ & Total \\
\hline Cereals(59) & 1534.48 & 3.19 & 1.64 & 0 & 63.39 & 24.39 & 1627.09 \\
Fruits and Vegetables(55) & 1128.40 & 3.40 & 1.48 & 0.03 & 60.12 & 35.53 & 1228.96 \\
Milk Products(50) & 1204.29 & 2.90 & 8.38 & 0 & 54.27 & 23.49 & 1293.33 \\
Pulse BasedProducts (56) & 1823.14 & 3.81 & 12.17 & 0 & 84.23 & 41.47 & 1973.82 \\
Products(56) & & & & & & & \\
Eggs(3) & 966.91 & 3.38 & 0.68 & 0 & 89.96 & 25.21 & 1086.14 \\
Other Items(7) & 1167.18 & 32.29 & 1.01 & 0 & 66.76 & 16.76 & 1284 \\
Nuts(3) & 1420.17 & 19.2 & 1.25 & 0 & 47.55 & 32.2 & 1520.37 \\
Water\#(24) & 1.76 & 1.02 & 0 & 0 & 0.05 & 0.06 & 2.89 \\
\hline
\end{tabular}

$\# \mu \mathrm{g} / 100 \mathrm{ml}$, Figures in parenthesis indicate the number of samples analyzed. 
cereals, fruits and vegetables and nuts were similar in home based and institutionalized diets.

\section{The mercury content of diets was negligible}

Food groups namely cereal and cereal based products, fruits and vegetables, milk and milk products, pulse based products and other items had high nickel contamination in the home based diets as compared to the diets of institutionalized subjects of the study population (Figure 4). However in eggs and nuts nickel contamination was detected to be higher in the diets of institutionalized subjects. In eggs the nickel contamination was two times higher in institutionalized diets as compared to the home based diets.

Institutional diets were found to have higher levels of Lead in cereals, pulses and eggs. Home based diets had higher lead content in fruits and vegetables, other items and nuts (Figure 5). However

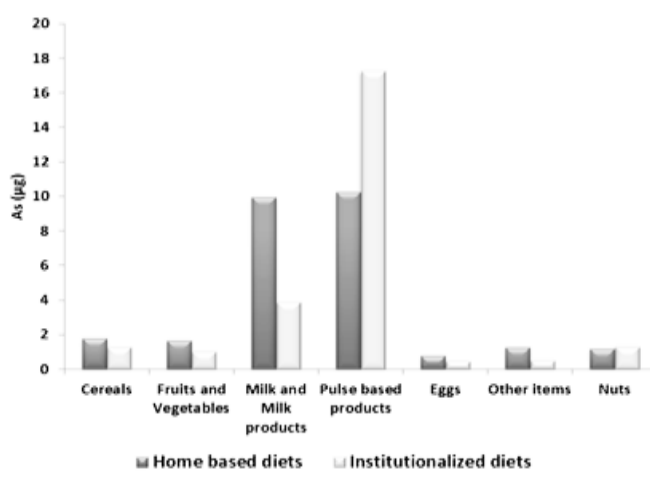

Fig. 3: Comparison of Concentration of Cadmium ( $\mu \mathrm{g} / 100 \mathrm{~g}$ of cooked food) in home based and institutionalized diets

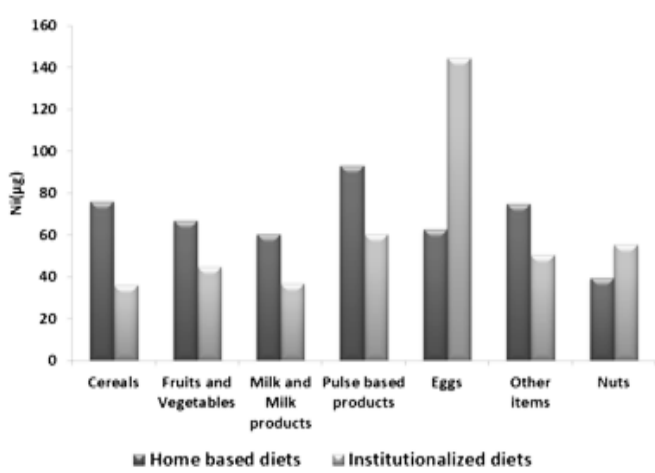

Fig. 4: Comparison of Concentration of Nickel ( $\mu \mathrm{g} / 100 \mathrm{~g}$ of cooked food) in home based and institutionalized diets milk and milk products from home based and institutionalized diets had similar levels of Lead.

It was observed that the Aluminum and Lead content was higher in the diets of institutionalized subjects. However Arsenic and Nickel content was higher in the home based diets of the study population. Cadmium level was found to be similar in both institutionalized and home based diets of the study population. High Aluminum content in the institutionalized diets can be attributed to the use of Aluminum utensils for cooking. Similar findings have been reported from a study from Switzerland that reported migration of Aluminum from cooking utensils into foods and beverages which was determined at intervals during cooking or during storage by graphite furnace atomic absorption spectroscopy. After 60 minutes of cooking in Aluminum pan an Aluminum content of $10-15 \mathrm{mg} /$ $\mathrm{kg}$ was measured in tomato sauce. The Aluminum concentration was also increased up to $2.6 \mathrm{mg} / \mathrm{l}$ after boiling tap water for 15 minutes in Aluminum pans (Müller, Steinegger, \& Schlatter, 1993). A study from UK found that Nickel leaching from stainless steel utensils contributes to $1 / 5$ th of the total dietary intake of Nickel therefore high levels of Nickel in the home based diets can be associated with the utensils used for cooking purpose (Flint \& Packirisamy, 1995).

\section{Health risk assessment with respect to Metal PTDI}

The provisional tolerable daily intake (PTDI) is the reference value, established by The joint FAO/ WHO expert committee on food additives (JECFA) , used to indicate the safe level of intake of a

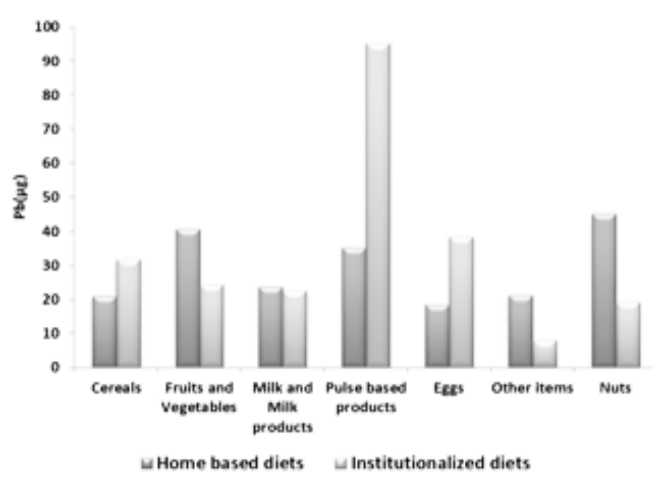

Fig. 5: Comparison of Concentration of Lead ( $\mu \mathrm{g} / 100 \mathrm{~g}$ of cooked food) in home based and institutionalized diets 
contaminant. The PTDI is calculated on a daily basis for contaminants which accumulate in the human body. The PTDI value obtained for the study population of the current study for Aluminum $(\mathrm{P}<0.05)$ and Lead $(P<0.001)$ were significantly higher than the PTDI cutoff. PTDI value for Aluminum and lead were $71.43 \%$ and $48.89 \%$ higher than the PTDI cutoff for the study population. The PTDI obtained for the study population of the current study for Arsenic and Mercury were significantly $(P<0.001)$ lower than the PTDI cutoff. The PTDI value for Arsenic and Mercury was $32.5 \%$ and $0.65 \%$ of the PTDI cutoff for the present study population of the present study. The PTDI value of Cadmium obtained for the study population was statistically non-significantly lower than the PTDI cutoff for Cadmium. The PTDI value of Cadmium estimated for the present study population was $74 \%$ of the PTDI cutoff for Cadmium.
TDI

The TDI value obtained for the study population for Nickel was significantly $(P<0.001)$ higher than the TDI cutoff for Nickel. The TDI value of Nickel for the study population was $104 \%$ higher than the TDI cutoff for Nickel.

Metal content in drinking water samples and comparison with US Environment Protection Agency (USEPA), World Health Organization (WHO) and Indian Standards Institute (ISI) guidelines.

It was observed that the levels of all the metals namely Aluminum, Arsenic, Lead and Mercury were much below the cutoff levels for the metals hence do not possesses any health risk to the study population.

\section{REFERENCES}

1. Khan S, Farooq R., Shahbaz S., Khan M. A., \& Sadique M, Health risk assessment of heavy metals for population via consumption of vegetables, 6(12), 1602-1606, (2009).

2. Zhuang P., McBride M. B., Xia H., Li N., \& Li Z, Health risk from heavy metals via consumption of food crops in the vicinity of Dabaoshan mine, South China. Science of the total environment, 10(061), 15511561,(2008).

3. Pandey J., \& Pandey U. Accumalation of heavy metals in dietary vegetables and cultivated soil horizon in organic farming system in relation to atmospheric deposition in a seasonally dry tropical region of India. Environ Monit Assess, 148: 61-74, (2009).

4. Shah M., \& Mehta A, Estimating the Environmental Cost of Industrialisation in Gujarat: A Case Study of Ankleshwar GIDC. Enviornmental Economics Research
Community,(2003)

5. Saipan P., \& Ruangwises S, determination of arsenic intake by duplicate diet portion sampling method, Journal of Enviornment and Research, 31(2): 25-33, (2009).

6. Raghuramulu N., Nair K. M., \& Kalyanasundaram (Eds.), A Manual of Laboratory Techniques (2 ed.). Hyderabad: National Institute of Nutrition, (2003).

7. Müller P. J., Steinegger A., \& Schlatter C, Contribution of aluminium from packaging materials and cooking utensils to the daily aluminium intake. Swiss Society of Applied and Analytical Chemistry. Switzerland, (1993).

8. Flint G. N., \& Packirisamy S, Systemic Nickel: the contribution by stainless- steel cooking utensils. Nickel Development Institute. Birmingham: Munksgaard. 